\title{
Scaling laws for breathing frequencies of solitary modes in the Zakharov equations
}

\author{
G. I. de Oliveira and F. B. Rizzato* \\ Instituto de Física-Universidade Federal do Rio Grande do Sul, Caixa Postal 15051, 91501-970 Porto Alegre, Rio Grande do Sul, Brazil
}

(Received 27 July 2001; published 17 December 2001)

\begin{abstract}
We analyze typical time scales resulting from the coupled dynamics of high- and low-frequency wave components in solitary solutions of the Zakharov equations. Linear stability analysis around the solitary modes suggests that adiabatic regimes may be obtained in the limit of high- and low-field intensities where the disparity of eigenfrequencies is large. Full simulations, however, reveal that adiabaticity arising from oscillatory motion can in fact be observed only over relatively short periods of time prior to noticeable radiation emission.
\end{abstract}

DOI: 10.1103/PhysRevE.65.016604

PACS number(s): 05.45.-a

\section{INTRODUCTION}

The purpose of the present paper is to investigate time scales associated with solitary wave dynamics of the Zakharov equations [1]. The Zakharov equations are presently recognized to provide one of the most complete models describing the coupling of high- and low-frequency waves in nonlinear environments. The model is particularly powerful in plasma physics, where the high- and low-frequency modes are, respectively, electron and ion acoustic waves [2]. The Zakharov equations are known to support steady solitary solutions that manifest themselves in the form of isolated or solitary lumps along the space. The set of equations is not of the integrable type and one cannot refer to the solitary lump solutions as solitons $[3,4]$. However, likewise solitons, the solitary modes can breath in the sense that if they are slightly displaced from their equilibrium shape, an oscillatory process accompanied by radiation emission can be observed around that equilibrium.

In more precise terms, the Zakharov equations describe the two-wave interaction of the slowly varying envelope of the high-frequency mode with the full low-frequency mode, so one can expect two frequencies or time scales characterizing the oscillatory process. In the appropriate limits, as we shall see, each frequency will be respectively associated with one of the interacting waves. In particular, this will help us to recover the known fact from plasma physics that when field amplitudes are sufficiently small, the low-frequency mode has a relatively high natural frequency and becomes enslaved to the amplitude fluctuations of the high-frequency mode which has a much lower natural frequency. Analysis of regimes with high-field intensities will show somewhat similar enslaving behavior.

If one wishes to calculate the frequency spectrum of the problem, perhaps the most direct way is to perform an analysis based on a variational principle called the average Lagrangian. We substitute ansatz solutions with a small number of dynamical variables in the full field Lagrangian, minimize the action with respect to these dynamical variables, and obtain a finite dimensional set of equations that can help to

\footnotetext{
*Email address: rizzato@if.ufrgs.br; tel.:+55 5133166470 ; fax:+55 5133167286
}

obtain some information on the problem, such as oscillatory frequencies of stable equilibria, growth rates of unstable equilibria, or other relevant quantities. Although several features are left aside by the procedure, such as radiative emission for instance, judicious care allows us to predict what will be seen in a full simulation.

The procedure has been used in a number of recent works, some of which are of interest here. In some cases, the variational method was used in the context of the nonlinear Schrödinger equation (NLS) alone and a single oscillatory frequency around equilibrium solitary solutions, compatible with full numerical simulations of the problem, was obtained $[5,6]$. More recently, the procedure was used in the context of the Zakharov equations [7], but then the frequency spectrum was not tested against full simulations. In any case, as one examines Ref. [7], one soon finds out that two frequencies are obtained as opposed to the single frequency obtained in the pure NLS case, which is a reasonable finding, but that both frequencies are always of the same order of magnitude-both proportional to the square of the highfrequency field amplitude. Now we would expect to see a more distinctive difference between these frequencies, such that one of them could become much smaller than the other in the known regime of very low intensities as mentioned above. One does not see that difference and this fact claims for a more detailed investigation of the problem. Also, the implications of distinct forms for both frequencies in regimes of high intensities should also be of relevance; we investigate the issue as well.

Given this framework, our plan is to rederive the simplified governing equations from an average Lagrangian principle, see if we can obtain the correct scaling laws for the frequency spectrum, analyze what is likely to survive when radiation effects are considered, and compare the results with full simulations of the Zakharov equations.

In Sec. II, we define the model, obtain the averaged equations, and determine scaling laws for the normal-mode frequency spectrum; in Sec. III, we compare the results obtained with full simulations, and in Sec. IV, we conclude the paper.

\section{THE MODEL, THE AVERAGE LAGRANGIAN, AND INITIAL ESTIMATES}

The set of Zakharov equations governing the nonlinear interaction of the envelope of high-frequency (HF) waves 
with low-frequency (LF) modes [2] read

$$
\begin{gathered}
i E_{t}+E_{x x}+2 n E=0, \\
n_{t t}-n_{x x}=-\left(|E|^{2}\right)_{x x} .
\end{gathered}
$$

$E(x, t)$ represents the envelope of the HF field and $n(x, t)$ the fluctuations associated with the LF mode. Note that, as mentioned in the Introduction, while Eq. (1) is an amplitude equation which no longer displays HF features, Eq. (2) is full and provides the nonintegrable ingredients.

Equations (1) and (2) admit the following steady-state solution

$$
\begin{gathered}
E_{s}(x, t)=\eta \operatorname{sech}(\eta x) e^{i \eta^{2} t}, \\
n_{s}(x)=\left|E_{s}\right|^{2},
\end{gathered}
$$

with $\eta$ as a positive but otherwise arbitrary amplitude parameter. The average Lagrangian method crucially depends on a well-done choice of Ansatz solutions. The Ansatz solutions should attend the following demands: resemble Eqs. (3) and (4) in the appropriate static limits; depend on a small number of time-dependent parameters; and be analytically treatable. In the present paper, we simply follow Ref. [7] and make the choice

$$
\begin{gathered}
E(x, t)=\sqrt{\frac{W_{e}}{a(t)}} e^{-x^{2} / 2 a(t)^{2}+i \phi(t)+i k(t) x^{2}}, \\
n(x, t)=\frac{W_{n}}{b(t)} e^{-x^{2} / b(t)^{2}},
\end{gathered}
$$

with $k(t)$ as the chirp factor. Constants $W_{e}$ and $W_{n}$ are, respectively, obtained from the conserved laws

$$
\begin{gathered}
W_{e} \equiv \frac{1}{\sqrt{\pi}} \int_{-\infty}^{+\infty}|E(x, t)|^{2} d x, \\
W_{n} \equiv \frac{1}{\sqrt{\pi}} \int_{-\infty}^{+\infty} n(x, t) d x,
\end{gathered}
$$

and are used to restrict the functional space over which we apply the method [7]. We also point out that from Eqs. (3) and (4) and from the properties of hyperbolic functions, the following equalities hold in equilibrium:

$$
W_{e}=W_{n}=\frac{2 \eta}{\sqrt{\pi}}
$$

a result to be used later. Note that the parameters

$$
a(t), b(t), \phi(t), k(t)
$$

may depend on time and define the reduced variational problem; i.e., those are the parameters whose dynamics we wish to study with the average method.
To obtain the appropriate governing equations, we first introduce the full Lagrangian controlling the coupled dynamics represented by Eqs. (1) and (2)

$$
\begin{gathered}
L=\int_{-\infty}^{+\infty} \mathcal{L} d x \\
\mathcal{L} \equiv \frac{i}{2}\left(E^{*} E_{t}-E E_{t}^{*}\right)-\left|E_{x}\right|^{2}+2 u_{x}|E|^{2}+u_{t}^{2}-u_{x}^{2} .
\end{gathered}
$$

$i^{2}=-1$, where $u(x, t)$ is an auxiliary variable defined according to $n(x, t)=u_{x}(x, t)$. Exact dynamical equations are to be obtained from

$$
\frac{\partial}{\partial t} \frac{\partial \mathcal{L}}{\partial g_{t}}+\frac{\partial}{\partial x} \frac{\partial \mathcal{L}}{\partial g_{x}}-\frac{\partial \mathcal{L}}{\partial g}=0,
$$

with $g=E(x, t), E(x, t)^{*}$, or $u(x, t)$, and our approximate form arises when we replace the exact fields with the Ansätz (5),(6), integrate Eq. (12) along space, and obtain the EulerLagrange equations for the remaining parameters. After some algebra, and focusing attention on the case $W_{e}=W_{n}$ $\equiv W$, we arrive at the following integrated form $L$ :

$$
\begin{aligned}
L= & -\frac{W}{2 a(t)^{2}}-\frac{W^{2}}{\sqrt{2} b(t)}+\frac{2 W^{2}}{\sqrt{a(t)^{2}+b(t)^{2}}}-2 W a(t)^{2} k(t)^{2} \\
& +\frac{W^{2} b(t)^{2}}{4 \sqrt{2} b(t)}-\frac{W a(t)^{2} k(t)}{2}-W \dot{\phi}(t) .
\end{aligned}
$$

Note that we have restricted the analysis to a situation where the constants of motion $W_{e}$ and $W_{n}$ have the same magnitude, a choice that does not affect the generic character of our final conclusions. All terms of the average Lagrangian (14) are similar to Eq. (14) of Ref. [7] with the exception of our term proportional to

$$
\dot{b}(t)^{2} / b(t),
$$

which appears in a form proportional to

$$
b(t) \dot{b}(t)^{2}
$$

there. We believe that our version agrees with some established properties of Zakharov equations and the reasoning comes in the following. We start by noting that to transform term (15) into a quadratic derivative with no dependence on a "coordinate" such as $b(t)$ so as to produce second temporal derivatives under the machinery of action principle, we shall rescale the field $b$ as $b(t) \rightarrow \beta(t) \equiv b(t)^{1 / 2}$, which is different than the rescaling used previously. As a direct consequence of this fact, our dynamical equations takes on the following aspect:

$$
\begin{aligned}
& \ddot{a}(t)=\frac{-8 W a(t)}{\left[a(t)^{2}+\beta(t)^{4}\right]^{3 / 2}}+\frac{4}{a(t)^{3}}, \\
& \ddot{\beta}(t)=\frac{1}{\beta(t)^{3}}-\frac{2 \sqrt{2} \beta(t)^{3}}{\left[a(t)^{2}+\beta(t)^{4}\right]^{3 / 2}} .
\end{aligned}
$$


$a=\beta^{2}=\sqrt{2} / W$ remains as the stable equilibrium, but the normal-mode frequencies around it now read

$$
\omega_{ \pm}^{2}=\frac{-\left[W^{2}\left(-3-5 W^{2}\right)\right] \pm \sqrt{-24 W^{6}+W^{4}\left(-3-5 W^{2}\right)^{2}}}{4}
$$

from which we can obtain simpler expressions in extreme limits

$$
\begin{gathered}
\omega_{+}=\sqrt{\frac{3}{2}} W, \omega_{-}=W^{2}, \text { if } W \ll 1, \\
\omega_{+}=\sqrt{\frac{5}{2}} W^{2}, \quad \omega_{-}=\sqrt{\frac{3}{5}} W, \text { if } W \gg 1 .
\end{gathered}
$$

We now point out that Eq. (1) relates a first-order time derivative with a second-order spatial derivative and that Eq. (2) relates time and space derivatives of the same order. If one associates the spatial derivative with the inverse size of the solitary solution $1 / L_{s}$ with $L_{s}$ satisfying $L_{s} \sim 1 / \eta \sim 1 / W$, then, if in addition one makes the replacements $\partial / \partial t$ $\rightarrow-i \omega_{e}$ and $-i \omega_{n}$ in Eqs. (1) and (2), respectively, one arrives at the following estimates:

$$
\omega_{e} \sim W^{2} \text { and } \omega_{n} \sim W .
$$

Frequencies (22) are not rigorous results, of course, but they nevertheless suggest that the linear and quadratic scalings obtained in Eqs. (20) and (21) are indeed what should be expected in terms of the general behavior associated with fields $E$ and $n$, respectivel: $\omega_{e}$, the frequency associated with oscillations of the $E$ field, bears direct relationship with $\omega_{-}\left(\omega_{+}\right)$when $W \ll 1 \quad(W \gg 1)$ whereas $\omega_{n}$, the frequency associated with oscillations of the $n$ field bears direct relationship with $\omega_{+}\left(\omega_{-}\right)$when $W \ll 1 \quad(W \gg 1)$. In particular, one would obtain the correct limit of $\omega_{n} \gg \omega_{e}$ for lowamplitude fields satisfying $W \ll 1$. We shall also see that in regimes with $W \gg 1$ the general scaling behavior can be also observed.

\section{FULL SIMULATIONS}

This much we can tell with the help of the averaged or reduced system. One could proceed and simulate the dynamics generated by the pair (17) and (18), but this would not provide much further insight on the problem. The fact is that the averaged equations are to be used with care and restrictions since they do not contain any reference to wave radiation. It is not guaranteed that the oscillatory modes just found and represented in relations (19) really persist as time evolves. Much to the contrary, one expects that at least some of the modes be relatively short lived as a result of wave radiation. However, the following is plausible. Consider the case $W \ll 1$. The steady solitary solution $E_{s}$, Eq. (3), contains an exponential factor of the form $e^{i \eta^{2} t}$. As explained in Ref. [8], this factor creates a gap of size $\eta^{2}$ in the continuous spectrum of the corresponding field such that direct decay of instabilities into the gap is not possible. According to our estimates, frequency $\omega_{-}$approaches $\eta^{2}$ when $W \ll 1$ with $\eta^{2} / \omega_{-} \sim 0.8$, which, although smaller than the unity, may inhibit radiation into this channel. As for the LF $n$ field, no exponential factor is present in $n_{s}$, Eq. (4), and fluctuations can freely decay into the continuum there. What we expect to see then is a rapid radiative decay of fluctuations associated with the $n$ field such that this LF field soon becomes enslaved to the slower motion associated with the envelope of the HF field $E$. Slower fluctuations of the $E$ field, however, tend to be more persistent in view of the inhibited direct decay into its continuum. Let us see if these features can be really captured in full simulations of set (1) and (2). We take a system of length $L$ centered at $x=0$ and extending from $x=-L / 2$ to $x=+L / 2$. The solitary solution is also centered at $x=0$ and has a much smaller length $L_{s} \ll L$-in the runs we use $L=40 L_{s}, L_{s} \equiv 2 / \eta$. Small dissipative terms are added to both $E$ and $n$ field equations and are turned on only when $|x|>10 L_{s}$ in order to extinguish emitted radiation. We use a finite difference method, and, independently, a Bulirsch-Stoer integrator combined with pseudospectral analysis; both techniques produce identical results. Our discretization uses $N=512$ (and 1024 to check convergence) grid positions and relative errors are inferior to a relative factor of $10^{-6}$. Finally, initial conditions are written down in a form satisfying definitions (7) and (8)

$$
\begin{gathered}
E(x, t=0)=\sqrt{\frac{W \pi^{1 / 2}}{2 a_{o}}} \operatorname{sech}\left(\frac{x}{a_{o}}\right), \\
n(x, t=0)=\frac{W \pi^{1 / 2}}{2 b_{o}} \operatorname{sech}^{2}\left(\frac{x}{b_{o}}\right),
\end{gathered}
$$

where parameters $a_{o}$ and $b_{o}$ are adjusted independently. $a_{o}$ $=b_{o}=2 /\left(\pi^{1 / 2} W\right)=1 / \eta$ corresponds to equilibrium and we will be using $a_{o}=1 / \eta \times 1.2$ and $b_{o}=a_{o} / 1.2$ for all initial conditions, so as to create an initial difference between $E$ and $n$.

In Fig. 1, we begin with the numerical analysis for small values of $W \ll 1$. Let us first take $W=0.1$ in panel (a) where time series for $|E(x=0, t)|^{2}$ and $n(x=0, t)$ are compared using a logarithmic scale for the time axis so as to comprise all relevant temporal scales of the problem in this one figure. We compute both fields right at the center of the breathing solitary waves and readily note the oscillatory pattern and the natural tendency of both fields to equalize after a short transient. The oscillatory pattern outliving the short transient is reasonably approximated by the smallest frequency $\omega_{-}$ $=W^{2} \sim \omega_{e}$ in this regime $W \ll 1$ and corresponds to oscillations of the $E$ field. We run the simulation up to $W^{2} t /(2 \pi)$ $=5$ and see approximately four oscillations in the $E$ component, which roughly means that as far as fluctuations in the $E$ field are concerned, $\omega_{-} \sim W^{2} \sim 1.2 \omega_{e, \text { sim. }}$. More finely tuned approximations obtained with the help of inverse scattering techniques improve the agreement between analytical estimates and simulations diminishing the factor of 1.2 in the relation above [6]. The $n$ field is largely over damped and the period of its existence as an independent entity actually de- 

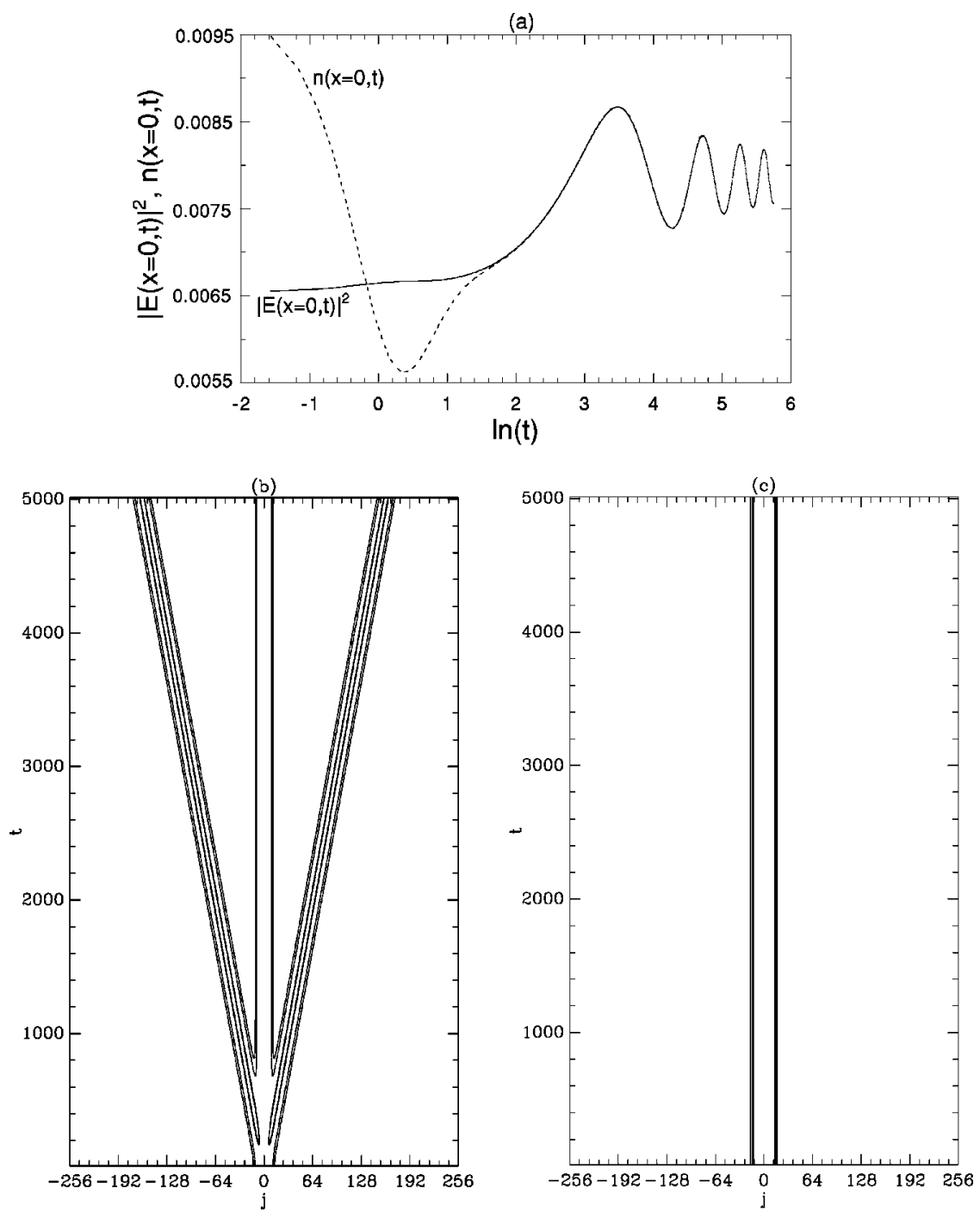

FIG. 1. $|E(x=0, t)|^{2}$ and $n(x$ $=0, t)$ versus time in panel (a), contour levels of $n(x, t)$ in (b), and contour levels of $|E(x, t)|^{2}$ in (c); all cases with $W=0.1$ and $j$ $\equiv N(x / L)$. fines what we call transient here. During equalization, radiation is emitted mostly into the $n$ field, as comparison of panels (b) [contour levels of $n(x, t)$ ] and (c) [contour levels of $\left.|E(x, t)|^{2}\right]$ shows; the lateral wings in panel (b) represent the emitted radiation and similar wings are absent in panel (c). Contour levels in both panels are drawn for field levels $|E(x, t)|^{2}, n(x, t)$ satisfying $0.1 W^{2} \gtrsim|E|^{2} \sim n \geqslant 0.0001 W^{2}$ so as to focus on the largest amplitude radiation emitted. At this point, we can be a little more emphatic and once again observe that equalization and radiation emission actually occur as $n(x, t)$ accommodates to the dynamics of $|E(x, t)|^{2}$. Therefore, $n$ does not keep oscillating as the average method predicts, but rapidly emits radiation into the continuum until the difference $|E|^{2}-n$ reduces substantially within the central region occupied by the solitary wave-subsequent oscillations are exclusively due to the dynamics of the $E$ field and are approximately governed by the NLS; all of this is represented in panels (a)-(c). Thus, there is not actually much sense in speaking about a well-established frequency $\omega_{n}$ $\sim \omega_{+}$here since the $n$ field is clearly over damped, but a much more reasonable feature to be measured is the scaling behavior determined earlier. Let us define the scaled time $\tau$ $\equiv W t$ and perform two runs for $W \ll 1$ so as to allow for the use of relations (20); one for $W=0.01$ and another for a larger value $W=0.1$. Given that the theoretically estimated fastest time scale [the period, as determined from Eq. (20)] is proportional to $1 / W$, if our scaling laws are correct, we expect to see similar figures in both cases since the horizontal axis already represents the scale-independent quantity $j$ $\equiv N(x / L)$ (let us recall that in our simulations, coordinates scale with the solitary lump length $\sim 1 / W$ ). This will suggest that although we cannot define a proper frequency for the fast time scale, the quantity $\omega_{+} \sim W$ from Eq. (20) may still be helpful, but now as a factor measuring the size of this fast temporal scale. The comparison can be seen in panels (a) and (b) of Fig. 2 from which, as predicted, the figures turn out to be quite similar.

When $W \gg 1$, the situation undergoes some significant changes as displayed in Fig. 3. In terms of order-ofmagnitude, the roles of frequencies $\omega_{e}$ and $\omega_{n}$ are reversed and now $\omega_{e} \gg \omega_{n}$. Figure 3(a), where we take $W=10$, confirms what we expect: the $E$ field rapidly oscillates about a slowly varying background determined by the relatively slow dynamics of the $n$ field; similarly to panel (a) of Fig. 1, we compare both fields evaluated at $x=0$, with time again rep- 

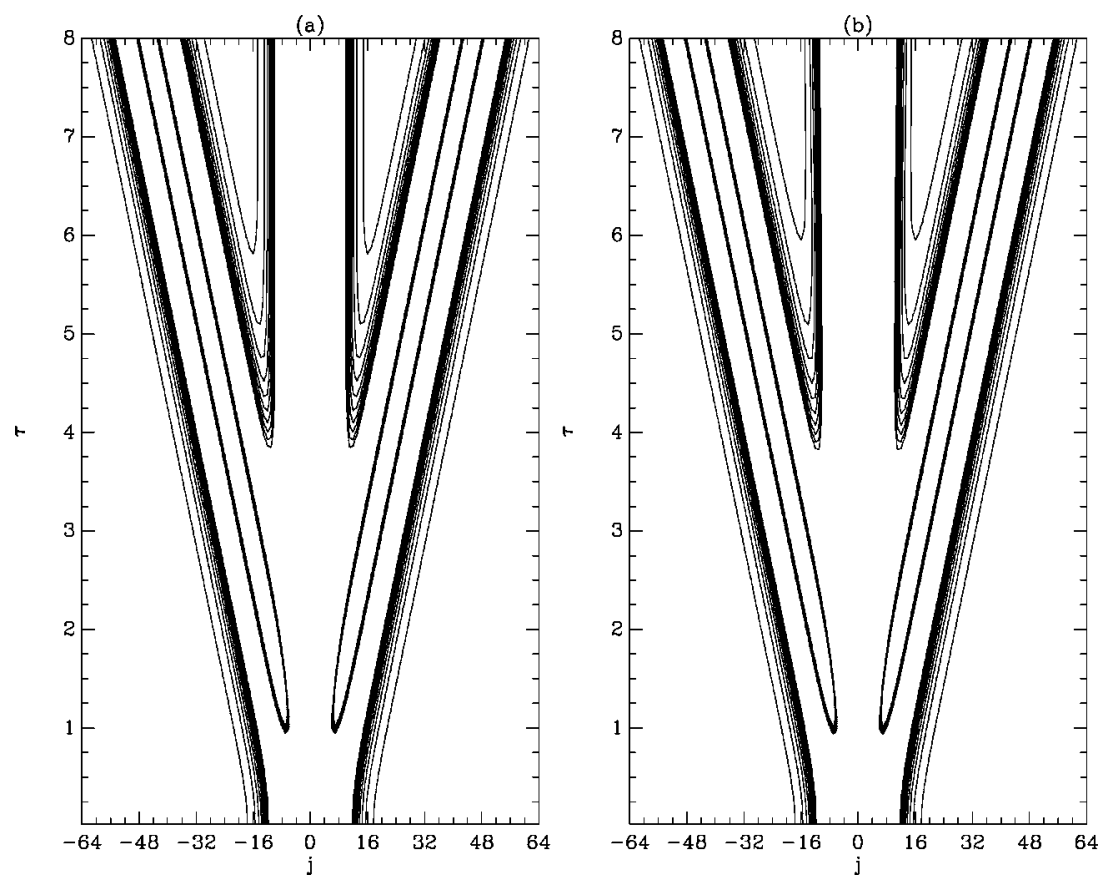

FIG. 2. Contour levels of $n(x, t)$ for two small values of $W$; $W=0.01$ in (a) and $W=0.1$ in (b). Again, $j \equiv N(x / L)$ and now $\tau$ $\equiv W t$.
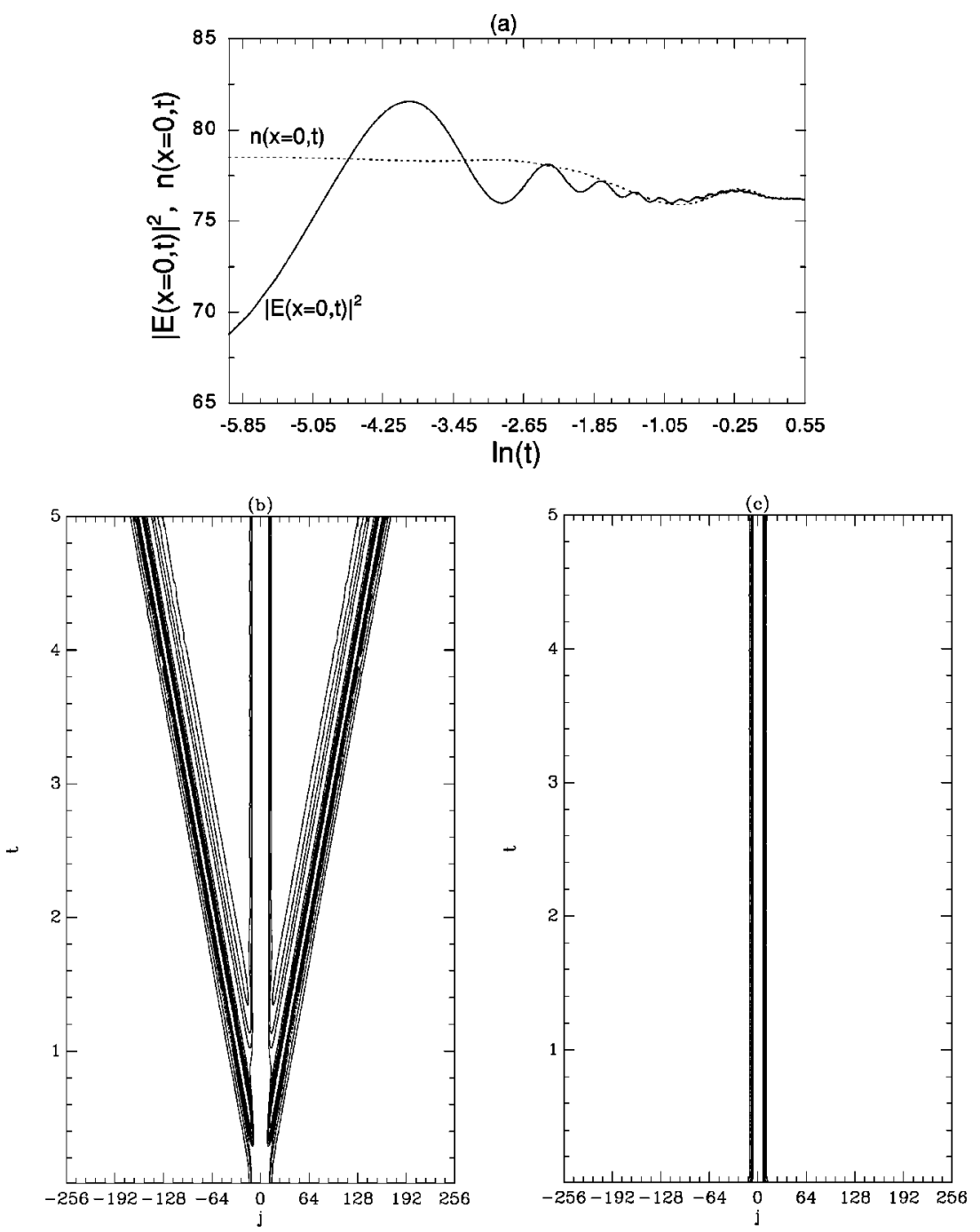

FIG. 3. The same set of panels as in Fig. 1, but now for $W=10$. 


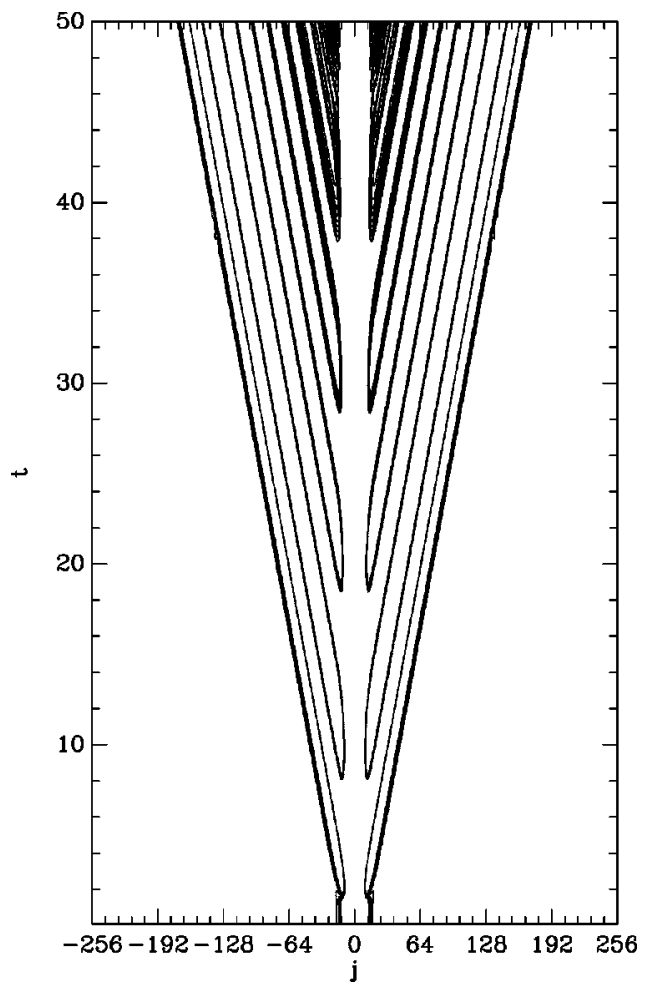

FIG. 4. Contour levels of $n(x, t)$ now for an intermediary value of $W, W=1$; again, $j \equiv N(x / L)$.

resented on a logarithmic scale. Within a small number of oscillations, certainly much smaller than the number of oscillations executed by the $E$ field either here or in the previous case $W \ll 1, n$ decays into an equilibrium state. Again, emission into the continuum of $E$ is much weaker than emission into the continuum of $n$. This is once more revealed when contour levels of $n(x, t)$ and $|E(x, t)|^{2}$ are compared in panels (b) and (c). Lateral wings, i.e., radiation, may be seen only in panel (b). The much larger number of oscillates of the $E$ field of panel (a) already suggested this kind of behavior; in other words, the $E$ field can once more be seen as a persistent oscillator similar to an internal mode of the soliton. For larger values of $W$ such as $W=10$ investigated here, and examining the dynamics of $E$, disagreement involving simulations and analytical predictions becomes more pronounciated than in the $W \ll 1$ case. In our case $W=10$ we observe that in regard to oscillations of the $E$ field, $\omega_{+}$ $\sim \sqrt{5 / 2} W^{2} \sim 1.5 \omega_{e, \text { sim. }}$. On the other hand, now the $n$ field does not decay as fast as in the case of small amplitude modes - this is suggested by Fig. 3(a) — and we can run additional simulations to have the following estimate $\omega_{-}$ $\sim \sqrt{3 / 5} W \sim 1.05 \omega_{n, \text { sim. }}$, which reveals a much better agreement; we do not show it here, but scaling laws inspired in Eqs. (21) still prevail.

We finally analyze the case $\omega_{e} \sim \omega_{n}$ taking $W=1$. Now the dynamics is highly nonadiabatic in the sense that there is no slow and fast time scales. The $n$ field still dissipates much more radiation than the $E$ field, but now, due to the increased resonance in the interaction, $n$ takes longer to settle down and reach the equilibrium. Figure 4 actually shows that radiation is emitted from the $n$ solitary wave not as single pulse as before, but as a continued collection of pulses. Further simulations show once again radiative wings cannot be observed in association with the $E$ field.

\section{Final conclusions}

In the present paper, we investigate how the solitary wave dynamics generated by the Zakharov equations behaves as a function of the system energy. Following an average Lagrangian approach, the $n$ and $E$ component of the solitary wave are let to vary independently, from which we extract two frequencies characterizing fluctuations around the static solitary equilibrium. The frequencies scale with energy in a different fashion than previous results predict, which allows us to reach adiabatic regimes both in small and large energy cases; previous works predict equal scaling laws for the two frequencies, and hence, the absence of adiabaticity. Then, as we perform full simulations of the nonlinear system, we note that the frequency associated with the $n$ field does not survive long; the respective fluctuations rapidly decay into the radiative continuum and do not remain confined within the solitary wave region. This means that this frequency cannot be seen as an internal mode of the solitary wave and must be reinterpreted. It is reasonable to see it as a quantity yielding a measure of the respective time scale. In the small energy case $W \ll 1$, we confirm that the emission process behaves in agreement with the time scale defined by $\omega_{n}$. In addition, once the $n$ component of the solitary wave radiates, it simply accompanies the fluctuations of the $E$ field and this is exactly what is to be expected in such a subsonic regime approximately described by a NLS equation.

In cases of large energies with $W \gg 1$, the situation is partially reversed: now the typical frequencies satisfy $\omega_{n} \ll \omega_{e}$, although radiation emission from the $n$ component into the continuum remains larger. We again note that oscillations in the $E$ field can be approximately seen as a normal internal mode, while oscillations in the $n$ field are damped in a faster way.

In intermediary cases $W \sim 1$, adiabaticity is lost. The key result of the increased resonant interaction between $n$ and $E$ fields is the larger number of oscillates of $n$. Radiation in this case is not confined to a single pulse, but is more uniformly distributed along the temporal axis as seen in Fig. 4 where we consider specifically $W=1$. Relaxation takes longer to happen here. $E$ is still weakly radiating and $n$ radiates more persistently than in adiabatic regimes.

\section{ACKNOWLEDGMENTS}

This work was partially supported by Conselho Nacional de Desenvolvimento Científico e Tecnológico (CNPq), Brazil. Some of the numerical computing was performed on the CRAY Y-MP2E at the Universidade Federal do Rio Grande do Sul Supercomputing Center. 
[1] V. E. Zakharov, Zh. Eksp. Teo. Fiz 62, 1745 (1972) [Sov. Phys. JETP 35, 908 (1972)].

[2] S. G. Thornhill and D. ter Haar, Phys. Rep. 43, 43 (1978).

[3] X. He and C. Zheng, Phys. Rev. Lett. 74, 78 (1995).

[4] G. I. Oliveira, L. P. L. Oliveira, and F. B. Rizzato, Phys. Rev. E 54, 3239 (1996).

[5] D. Anderson, Phys. Rev. A 27, 3135 (1983).
[6] E. A. Kuznetsov, A. V. Mikhailov, and I. A. Shimokhin, Physica D 87, 201 (1995).

[7] B. Malomed, D. Anderson, M. Lisak, M. L. Quiroga-Teixeiro, and L. Senflo, Phys. Rev. E 55, 962 (1997).

[8] Y. S. Kivshar and B. Malomed, Rev. Mod. Phys. 61, 763 (1989). 